Artigo Original

\title{
Exposição crônica em ambiente de conflito sensorial e sua influência no controle postural ${ }^{1}$
}

\author{
Danielle Brandalize ${ }^{1}$ \\ André Luiz Felix Rodacki ${ }^{2}$ \\ Michelle Brandalize \\ Vera Lúcia Israel ${ }^{2}$ \\ ${ }^{1}$ Curso de Fisioterapia, Faculdade Guairacá, Guarapuava, PR, Brasil \\ ${ }^{2}$ Programa de Pós-Graduação em Educação Física, \\ Universidade Federal do Paraná, Curitiba, PR, Brasil
}

\begin{abstract}
Resumo: O objetivo do presente estudo foi investigar o equilíbrio de indivíduos expostos cronicamente a um ambiente com conflito sensorial, antes e imediatamente após a exposição a este ambiente. Quinze pescadores participaram voluntariamente e tiveram seu deslocamento do centro de pressão (CP) mensurado por uma plataforma de força, antes e após a jornada no mar. Seis diferentes condições, nas quais as informações sensoriais dos sistemas visual e somatossensorial foram manipuladas, foram testadas. A trajetória total do centro de pressão indicou a habilidade dos participantes em utilizar cada sistema sensorial separadamente, em cada condição para o controle postural. Não houve alterações na oscilação corporal ou na dependência sensorial imediatamente após a exposição ao ambiente com conflito sensorial. Uma adaptação crônica parece evitar com que eles sejam influenciados pelos efeitos agudos da exposição a um ambiente com conflito sensorial.
\end{abstract}

Palavras-chave: Equilíbrio postural. Modalidade sensorial. Pesqueiros.

\section{Chronic exposure in an environment of sensory conflict and its influence on postural control}

\begin{abstract}
The aim of the present study was to evaluate the balance of subjects chronically exposed to an environment with sensorial conflict before and immediately after exposition to this environment. Fifteen fishermen volunteered to participate and had their displacement of center of pressure (CP) measured by a force platform before and after a sea journey. Six different conditions, in which the sensorial information of visual and somatosensory system was manipulated, were tested. The total path of the center of pressure indicated the participant's ability to use each sensory system separately for each condition to control upright posture. There were no changes in body sway or sensory dependence immediately after exposure to an environment with sensorial conflict. A chronic adaptation seemed to prevent them to be influenced by acute effects of exposure to an environment with sensorial conflict.
\end{abstract}

Keywords: Postural balance. Modalities sensorial. Fisheries.

\section{Introdução}

O controle postural envolve uma íntima relação entre as informações sensoriais e os comandos motores (POLLOCK et al., 2000). As informações dos sistemas vestibular, visual e somatossensorial convergem para o sistema nervoso central, onde são integradas num processo contínuo e dinâmico que permite obter informações acuradas sobre a configuração do corpo no espaço para a seleção de respostas motoras adequadas (HORAK; MCPHERSON, 1996; JEKA et al., 2000; KAUFMAN et al., 2001).

As informações sensoriais usadas pelo sistema de controle postural para manter 0

\footnotetext{
1 Trabalho derivado de dissertação de mestrado realizado pelo Programa de Pós-Graduação em Educação Física da Universidade Federal do Paraná.
}

equilíbrio do corpo dependem, em parte, das informações disponíveis no ambiente. Em condições nas quais não há conflito sensorial, as informações provenientes dos sistemas, visual, somatossensorial e vestibular serão congruentes (MAHBOOBIN et al., 2009). Todavia, conflitos sensoriais podem ocorrer quando as informações dos sistemas sensoriais forem incongruentes (REASON, 1978) como ocorre, por exemplo, durante a locomoção passiva em automóveis, ônibus, barcos ou aviões, nessas condições, o sistema vestibular indica movimento e o visual não, uma vez que o indivíduo esteja olhando para dentro do meio de transporte.

Portanto, em ambientes onde há conflito de informações é fundamental que o sistema nervoso central seja capaz de selecionar a 
informação que possa representar mais proximamente a verdadeira orientação corporal, a fim de que uma resposta adequada possa ser gerada (HORAK; SHUPERT, 1994). Dependendo do contexto ambiental onde a tarefa é realizada, o sistema de controle postural atribui maior peso em um tipo de informação sensorial, dependendo quão útil é a informação para o sistema de controle postural (PETERKA, 2002; BONFIM et al., 2006; MAHBOOBIN et al., 2009).

Essa influência da informação sensorial sobre o controle postural tem sido investigada através da manipulação da informação de um ou mais sistemas sensoriais. Alguns estudos (NACHUM et al., 2004; BARELA et al., 2006, PARIRTTIWINKLER et al., 2008) manipularam as informações sensoriais dos sistemas visual, somatossensorial e/ou vestibular para avaliar a capacidade do indivíduo em utilizar as informações disponíveis no ambiente e de suprimir as informações imprecisas oriundas de cada um desses três sistemas.

Durante situações de conflito sensorial, alguns indivíduos apresentam dificuldade em selecionar a informação mais apropriada e desenvolvem um conjunto de sintomas: enjoo, náuseas, vômitos e tontura. Tais sintomas são chamados de cinetose (REASON, 1978). Quando esses sintomas são sentidos após a exposição ao conflito sensorial, denomina-se mal de desembarque. $\mathrm{O}$ mal de desembarque refere-se à ilusão de sensação de movimento após a exposição ao conflito sensorial e ocorre durante viagens em barcos, trens ou aviões (MOELLER; LEMPERT, 2007).

Shahal et al. (1999), investigaram o efeito agudo da cinetose e Nachum et al. (2004), analisaram 0 efeito agudo do mal de desembarque na organização sensorial do controle da postura ereta, em membros de uma tripulação naval. Ambos os estudos, observaram que a influência das informações vestibulares e visuais foi minimizada e que os indivíduos mais susceptíveis a desenvolverem cinetose e mal de desembarque eram, principalmente, dependentes das informações somatossensoriais para a manutenção do equilíbrio.

Alterações na dependência sensorial também foram encontradas em astronautas após voos espaciais, sendo que esses indivíduos, expostos à microgravidade (BLACK; PALOSK, 1998). Após a privação da gravidade uma maior confiança nas dicas de orientação visual e uma preferência pela informação somatossensorial foi observada para manter o equilíbrio e a estabilidade postural. Tais achados são compatíveis com o tipo de conflito aplicado, visto que o sistema vestibular se referencia pela força da gravidade.

Assim, a integração das informações sensoriais parece ser dinamicamente regulada para adaptar-se às mudanças nas condições ambientais e à informação sensorial disponível (HORAK; MCPHERSON, 1996). Durante ou logo após uma experiência de conflito sensorial, o sistema de controle postural se adapta mudando a importância dada às informações de cada sistema sensorial. Contudo, acredita-se que com exposição crônica a um ambiente com conflito sensorial, possa ocorrer uma adaptação permanente no sistema de controle postural. Os pescadores estão constantemente expostos a conflitos sensoriais e podem apresentar características ímpares que permitem entender o funcionamento do sistema de controle postural. Por isso, o objetivo do presente estudo foi avaliar a oscilação corporal em diferentes situações de conflito sensorial, em pescadores, antes e após sua jornada diária no mar. Com os resultados do presente estudo será possível demostrar se o sistema de controle postural pode sofrer uma modificação permanente consequente do estímulo repetido e assim, verificar a importância para a reabilitação de pessoas com disfunção em um dos sistemas sensoriais. E dessa forma, investigar o equilíbrio de indivíduos expostos cronicamente a um ambiente com conflito sensorial, antes e imediatamente após a exposição a este ambiente.

\section{Materiais e métodos}

\section{Participantes}

Pescadores cadastrados na Colônia de Pescadores Z-4 de Matinhos - PR foram convidados a participar voluntariamente do estudo. A amostra foi intencional e não randomizada, mediante a conveniência obtida para o contato com os participantes. Por meio de uma entrevista foram excluídos aqueles que apresentassem diagnóstico ou sintoma de algum tipo de alteração do equilíbrio ou qualquer queixa de comprometimento musculoesquelético que pudesse comprometer a realização das tarefas; desordem neurológica (Acidente Vascular Encefálico, Parkinson, Esclerose Múltipla, entre outros); sensorial e/ou motor (Lesão de nervo periférico, Diabetes, Distrofias, entre outras), 
assim como a ingestão de bebida alcoólica nas últimas 24 horas antes das avaliações.

Dessa forma, depois de aplicados os critérios de exclusão, 15 pescadores com idade média de $37,4( \pm 9.9)$ anos, média de massa corporal de $80,6( \pm 16,4) \mathrm{Kg}$ e estatura média de 172,2 $( \pm 7)$ $\mathrm{cm}$ foram selecionados. Todos assinaram um termo de consentimento livre e esclarecido para participação no estudo. $O$ termo de consentimento foi aprovado pelo Comitê de Ética do Setor da Saúde Universidade Federal do Paraná (Protocolo $n^{0}$ 752.087.09.07). Os pescadores eram expostos às atividades de pesca 5 dias por semana, durante 4 horas por dia. Os participantes tinham experiência média de $22,4( \pm 10,3)$ anos com a pesca.

\section{Procedimentos e instrumentos}

Inicialmente, os participantes responderam o Questionário de Susceptibilidade a Cinetose (GOLDING, 1998) para identificar e classificar indivíduos com maior predisposição a desenvolver sintomas. Os resultados foram utilizados para controle, visto que alguns estudos (SHAHAL et al., 1999; NACHUM et al., 2004) mostraram que essa é uma variável que pode influenciar as estratégias de equilíbrio. $O$ questionário é uma tradução (não validada) do "Motion sickness susceptibility questionnaire" revisado por Golding (1998). O questionário é dividido em duas sessões. A sessão "A" é referente às experiências do indivíduo com viagens e desenvolvimento de cinetose na infância, considerada antes dos 12 anos de idade. A sessão "B" refere-se às experiências em viagens e desenvolvimento de cinetose nos últimos 10 anos. A pontuação foi calculada como recomendado por Golding (1998) e varia entre 0 a 200 , sendo que "0" ponto indica menor susceptibilidade e 200, maior susceptibilidade a cinetose.

Testes de equilíbrio foram realizados em um único dia antes e após a jornada no mar, que na ocasião do teste durou em média 3 horas para cada sujeito. Os participantes foram instruídos a permanecerem na postura ereta e quieta com os pés juntos e os membros superiores ao lado do tronco, sobre uma plataforma de força, em 6 condições experimentais sobre uma superfície de apoio firme: com olhos abertos (C1), com olhos fechados (C2) e com informação visual imprecisa (C3) e sobre uma superfície de apoio instável (espuma): com olhos abertos (C4), com olhos fechados (C5) e com informação visual imprecisa (C6) (SHUMWAY-COOK; HORAK, 1986).
Nas condições em que os participantes permaneceram com olhos abertos, os mesmos foram orientados a olhar para um ponto fixo, posicionado a 2 metros de distância, na altura dos olhos. Uma espuma com densidade de $33 \mathrm{Kg} / \mathrm{m}^{3}$ e 10 centímetros de altura foi colocada sobre a superfície da plataforma de força (AMTI, modelo OR6-7-2000) para reduzir a precisão da informação somatossensorial (C4, C5 e C6). Uma cúpula foi empregada para fornecer informação visual imprecisa em C3 e C6. Esta cúpula foi construída como sugerido por Shumway-Cook e Horak (1986), utilizando-se uma lanterna japonesa branca, com $40 \mathrm{~cm}$ de diâmetro e uma circunferência de $270^{\circ}$. Tiras de fita preta foram colocadas no interior da cúpula formando linhas verticais afastadas entre si $2.5 \mathrm{~cm}$ na borda e 7 $\mathrm{cm}$ na região central. Um " $X$ " foi colocado dentro da cúpula para minimizar cansaço nas vistas. Depois de colocada na cabeça, a cúpula de conflito visual move-se em fase com os movimentos da cabeça. Os olhos ficam cerca de $20 \mathrm{~cm}$ de distância da parte anterior da cúpula, sendo que a visão periférica fica restrita nos aspectos superior, inferior e lateral. A Figura 1 apresenta a cúpula empregada no estudo.

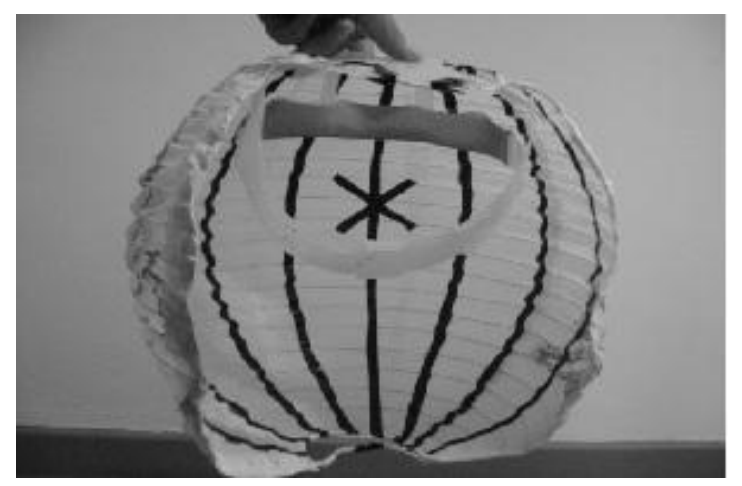

Figura 1. Cúpula para causar informação visual imprecisa.

Cada condição do teste teve duração de 40 s, dos quais os primeiros 10 s foram destinados a permitir que os participantes se acomodassem sobre a plataforma e a fim de minimizar possíveis efeitos de aprendizagem. $O$ registro dos dados foi realizado nos 30 s seguintes. Um intervalo de 30 s foi imposto entre cada condição experimental. Cada condição experimental foi testada uma única vez, em ordem aleatória.

Os deslocamentos do centro de pressão (CP) foram registados com uma plataforma de forças $A$ plataforma foi colocada em uma sala isolada, sobre uma superfície plana e nivelada sobre um tapete de borracha. Os dados foram amostrados com uma frequência de $100 \mathrm{~Hz}$. 


\section{Tratamento dos dados}

A partir dos deslocamentos do centro de pressão $(\mathrm{CP})$ a trajetória total, considerando-se o comprimento total da trajetória do $\mathrm{CP}$ nas direções anteroposterior e médio-lateral foi calculada. A trajetória total foi selecionada como variável deste estudo por representar por representar o deslocamento global dos sujeitos. Os dados foram filtrados por um filtro passa baixa de $10 \mathrm{~Hz}$ e analisados por programa específico escrito em ambiente de programação MatLab 7.0.

Para analisar a influência de cada sistema sensorial na manutenção da postura ereta, utilizou-se a razão do valor da trajetória total entre as condições $\mathrm{C} 2, \mathrm{C} 4$ e $\mathrm{C} 5$ pela condição $\mathrm{C} 1$. A capacidade do indivíduo em usar a informação somatossensorial foi calculada por C2/C1, para calcular a capacidade de usar a informação visual para manter o equilíbrio, utilizou-se a razão C4/C1 e para a capacidade em utilizar o sistema vestibular C5/C1. A habilidade em usar as informações visuais e somatossensoriais, mesmo imprecisas é indicada pela razão de $\mathrm{C} 3+\mathrm{C} 6 /$ C2+C5 (PARIETTI-WINKLER et al., 2008). A contribuição de cada sistema sensorial foi expressa em percentual de $\mathrm{C} 1$, portanto, quanto maior a razão entre elas, maior é a oscilação e, consequentemente, pior a capacidade em obter informações daquele determinado sistema.

\section{Tratamento estatístico}

Primeiramente, a normalidade da distribuição dos dados (Shapiro-Wilk) foi testada. Os resultados dos testes de normalidade indicaram a necessidade do uso de estatística não paramétrica. Portanto, o teste de Wilcoxon foi aplicado para identificar diferenças entre pré e pós-exposição ao conflito sensorial (jornada no mar) sobre a trajetória total e saber a razão da trajetória entre condições. Em todas as análises o nível de significância adotado foi de 0,05 e o programa Statistica (Statsoft, versão 7.0) foi utilizado para o tratamento estatístico.

\section{Resultados}

O questionário de susceptibilidade à cinetose não apontou susceptibilidade para desenvolver tais sintomas em nenhum dos participantes $(p>0,05)$. A maioria dos indivíduos não se considerou uma pessoa susceptível à cinetose $(91,6 \%)$, enquanto $8,4 \%$ consideraram-se pouco susceptível. A média da pontuação obtida no questionário de susceptibilidade à cinetose foi de 7,92 (variação 0 a 10,8).

Não foram encontradas diferenças na trajetória total de deslocamento do CP antes (PRE) e após (POS) a exposição ao conflito sensorial para as 6 condições experimentais $(p>0,05)$. Os valores médios e desvio padrão $( \pm d p)$ das variáveis estabilométricas são apresentados na Figura 2.

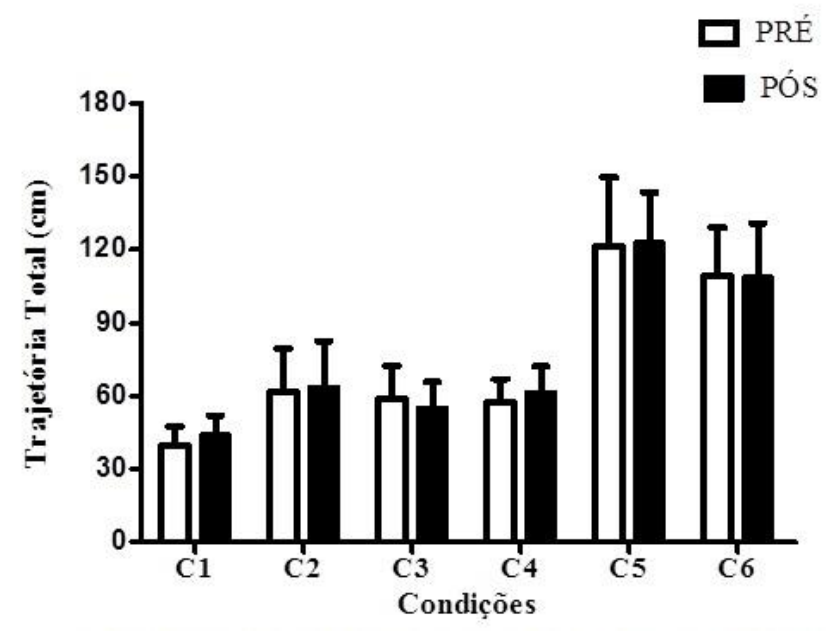

Legenda: (C1) Superfície de apoio firme e olhos abertos. (C2) Superfície de apoio firme e olhos fechados. (C3) Superfície de apoio firme e visão imprecisa. (C4) Superfície de apoio instável e e olhos abertos. (C5) Superfície de apoio instável e e olhos fechados. (C6) Superfície de apoio instável e visão imprecisa.

Figura 2. Valores da trajetória total de deslocamento do CP nas 6 condições experimentais, pré e pós exposição ao conflito sensorial.

A razão entre a trajetória de deslocamento do CP obtido em cada condição (C2, C4 e C5) pela condição $C 1$ não diferiu $(p>0,05)$ entre as avaliações pré e imediatamente após a exposição a um ambiente com conflito sensorial. A razão entre $\mathrm{C} 3+\mathrm{C} 6 / \mathrm{C} 2+\mathrm{C} 5$ dos testes realizados antes (PRE) e imediatamente após a exposição ao conflito sensorial também não diferiu $(p>0,05)$. $\mathrm{Na}$ Figura 3, esses resultados são expressos em percentual de $\mathrm{C} 1$. 


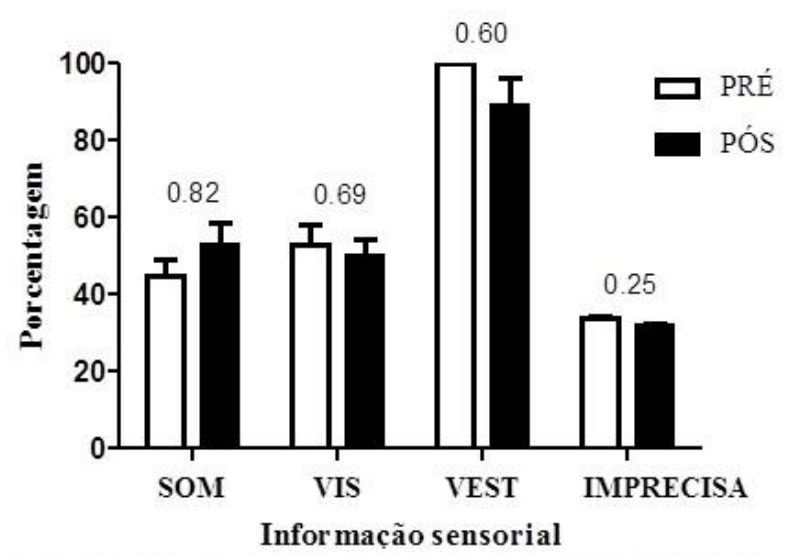

Figura 3. Valores percentuais da contribuição de cada sistema sensorial para a manutenção do equilíbrio e os valores de $p$ da comparação entre as avaliações pré e pós exposição ao conflito sensorial.

\section{Discussão}

Este estudo teve por objetivo investigar a influência da exposição a um conflito sensorial sobre a estabilidade postural e a preferência sensorial de pescadores. Nenhum dos participantes apresentou susceptibilidade para desenvolver cinetose ou mal de desembarque. Provavelmente, as adaptações decorrentes do prolongado tempo que os navegadores são expostos a esse tipo de estímulo podem ter reduzido os sintomas característicos desse conflito (REASON, 1978). Logo, a ampla experiência dos participantes (22,4 anos) pode ter influenciado esses resultados. De fato, outros estudos tem mostrado que adaptações à cinetose e à síndrome do desembarque ocorrem após um período de 22 meses (SHAHAL et al., 1999), os participantes desta pesquisa apresentaram uma experiência mínima de 6 anos.

Os resultados não indicaram alterações na oscilação corporal em função da exposição ao conflito sensorial. Desta forma, parece que a maior instabilidade postural durante uma situação de conflito sensorial, ou mesmo após a ela, está associada a maior susceptibilidade para desenvolver cinetose ou mal de desembarque (OWEM et al., 1998; SHAHAL et al., 1999; NACHUM et al., 2004). Uma segunda possibilidade para explicar a estabilidade postural frente ao conflito sensorial pode estar no período relativamente curto em que o estímulo foi aplicado (O'CONNOR et al., 2008). Por exemplo, alguns estudos tem reportado instabilidade postural após uma situação de conflito sensorial em que os participantes foram expostos por vários dias ao conflito (SHAHAL et al., 1999; NACHUM et al., 2004). Logo, parece que três horas de exposição pode não ter sido suficiente para causar maiores alterações sobre o equilíbrio corporal.

Existem indicativos de que quanto maior a quantidade de sistemas sensoriais manipulados durante os teste maior é sua influência sobre o equilíbrio e consequentemente, maior será a oscilação do centro de pressão corporal (RUWER et al., 2005). Alguns estudos têm observado que a dificuldade na manutenção do controle postural é aumentada quando as informações de dois sistemas sensoriais estão ausentes e/ou manipulados (MANN et al., 2011).

No presente estudo, constatou-se que os indivíduos apresentaram maior dificuldade em manter o equilíbrio na condição em que a informação visual estava ausente $e$ a somatossensorial imprecisa (condição C5), sendo que a dependência do sistema vestibular para manter o equilíbrio era maior do que nas demais condições experimentais. Isso indica que os indivíduos precisam de uma base de apoio estável e das informações visuais para sustentar uma postura ereta quieta e estável. Tais achados são sugestivos de uma possível disfunção vestibular ou menor dependência dos inputs desse sistema. Nota-se também, que os participantes se adaptam melhor a situações nas quais a informação sensorial está imprecisa do que quando está ausente. Isso pode estar associado à habilidade do sistema nervoso em mudar discretamente a fonte principal de informação sensorial para controlar a postura (MCCOLLUM et al. 1996). Isso explica porque astronautas são mais dependentes das informações somatossensoriais e visuais do que das informações vestibulares para manter 0 equilíbrio (BLACK; PALOSK, 1998). Assim como no mar, os conflitos gerados pela exposição à 
microgravidade acarreta uma diminuição na importância dada às informações vestibulares, que são as afetadas tanto no mar quanto no espaço. Os pescadores possivelmente acabam suprimindo as informações vestibulares e confiando mais nas informações somatossensoriais e visuais que estão presentes e relativamente preservadas em alto mar (SHAHAL, et al., 1999, BLACK; PALOSKI, 1998).

Contudo, pode-se observar que não houve mudança na dependência sensorial imediatamente após a exposição ao conflito sensorial. Isso sugere que com a exposição crônica ao conflito sensorial parece ocorrer uma adaptação do sistema de controle postural, fazendo com que os indivíduos não sofram mais os efeitos agudos da exposição ao conflito sensorial, como cinetose, mal de desembarque ou mesmo, alteração na confiança nas informações sensoriais disponíveis. Logo, os pescadores são capazes de manter suas estratégias, independente de terem saído de uma situação de conflito ou não.

Para a confirmação desses achados é importante que outros estudos sejam realizados envolvendo uma maior amostra e um grupo controle composto por pessoas não expostas cronicamente ao conflito sensorial. Além disso, maiores períodos de exposição são necessários para determinar se períodos relativamente breves de exposição ao conflito sensorial podem influenciar a capacidade de controlar o centro de pressão corporal. Estudos prospectivos com avaliações repetidas poderão avaliar quando as adaptações sensoriais são assimiladas e se tornam estáveis.

\section{Conclusões}

A exposição crônica ao conflito sensorial parece ter acarretado uma adaptação a esse conflito. Tal adaptação parece ter sido favorecida pela utilização de informações de diferentes sistemas sensoriais. Não foi possível determinar se as adaptações crônicas podem ter influenciado a resposta aguda ao conflito sensorial ou se o tempo agudo de exposição foi relativamente pequeno para gerar um conflito sensorial suficiente para causar perturbações agudas sobre o equilíbrio. Além disso, sujeitos cronicamente expostos a conflitos sensoriais mostraram confiar mais nas informações de fontes somatossensorial e visual do que na informação vestibular.

\section{Referências}

BLACK, F.O.; PALOSKI, W.H. Computerized dynamic posturography: what have we learned from space? Archives of Otolaryngology Head Neck Surgery. Chicago. v.118, p 45-51, 1998. doi: http://dx.doi.org/10.1016/S0194$\underline{5998(98) 70009-9}$

BARELA, J. A; BONFIM, T. A.; POLASTRI, P. F. Efeito do toque suave e da informação visual no controle da posição em pé de adultos. Revista Brasileira de Educação Física e Esporte. São Paulo, v.20, n.1, p.15-25, 2006. Disponível em: http://www.revistasusp.sibi.usp.br/scielo.php?scrip $\mathrm{t}=\mathrm{sci}$ arttext\&pid=S1807-

$55092006000100002 \& \operatorname{lng}=$ pt\&nrm=iso\&tlng=pt. Acesso em: 23 jan. 2010.

GOLDING, J. F. Motion sickness susceptibility questionnaire revised and its relationship to other forms of sickness. Brain Research Bulletin. Phoenix. v. 47, n. 5, p.507-516, 1998. doi: http://dx.doi.org/10.1016/S0361-9230(98)00091-4

HORAK, F.B.; MACPHERSON, J.M. Postural orientation and equilibrium. In: ROWELL. L.B.; SHEPARD. J.T. eds. Handbook of physiology. New York. Oxford University Press. p.255-92, 1996.

HORAK, F.B.; SHUPERT, C.L. The role of vestibular system in postural control. In: HERDMAN, S. (ED). Vestibular Rehabilitation. New York: F.A Davis. p. 22-46, 1994.

JEKA, J.J.; OIE, K.S.; KIEMEL, T. Multisensory information for human postural control: integrating touch and vision. Experimental Brain Research. Berlin. v.134, n. 1, p. 107-125, 2000. doi: http://dx.doi.org/10.1007/s002210000412

KAUFMAN, G. D.; WOOD, S.J.; GIANNA, C.C.; BLACK, F.O.; PALOSKI, W.H. Spatial orientation and balance control changes induced by altered gravitoinertial force vectors. Experimental Brain Research. Berlin. v.137, n. 3, p.397-410, 2001. doi: http://dx.doi.org/10.1007/s002210000636

MAHBOOBIN, A.; LOUGHLIN, P.; ATKESON, C.; REDFERN, M. A mechanism for sensory reweighting in postural control. Medical and Biological Engineering and Computing. Stevenage. v. 47, n. 8, p. 921-929, 2009. doi: http://dx.doi.org/10.1007/s11517-009-0477-5

MAN, L.; KLEINPAUL, J.F.; TEIXEIRA, C.S.; MOTA, C.B. Influência dos sistemas sensoriais na manutenção do equilíbrio em gestantes.

Fisioterapia e Movimento. Curitiba. v. 24, n. 2, p. 315-325, 2011. Disponível em:

http://www2.pucpr.br/reol/index.php/RFM?dd1=47 14\&dd99=view. Acesso em: 23 jan. 2010.

MCCOLLUM, C.; SHUPERT, C.L.; NASHNER, L.M. Organizing sensory information for postural control in altered sensory environments. Journal 
of Theoretical Biology. London. v.180, n. 3, p. 257-70, 1996. doi:

http://dx.doi.org/10.1006/itbi.1996.0101.

MOELLER, L.; LEMPERT, T. Mal de debarquement: Pseudo-hallucinations from vestibular memory? Journal of

Neurophysiology. Bethesda. v. 254, n. 6, p. 813815, 2007. doi: http://dx.doi.org/10.1007/s00415006-0440-4

NACHUM, Z.; SHUPAK, A.; LETICHEVSKY, V.; BEN-DAVID, J.; TAL, D.; TAMIR, A.; TALMON, Y.; GORDON, C. R.; LUNTZ, M. Mal de debarquement and posture: reduced reliance on vestibular and visual cues. The Laryngoscope. St. Louis. v.114, n.3, p.581-586, 2004. doi: http://dx.doi.org/10.1097/00005537-200403000$\underline{00036}$

O'CONNOR, K.W.; LOUGHLINA, P.J.; REDFERNA, M.S.; SPARTO, P.J. Postural adaptations to repeated optic flow stimulation in older adults. Gait Posture. Oxford. v. 28, n.3, p. 385-39, 2008. doi: http://dx.doi.org/10.1016/j.gaitpost.2008.01.010

OWEN, N.; LEADBETTER, A.G.; YARDLEY, L. Relationship between postural control and motion sickness in healthy subjects. Brain Research Bulletin. Phoenix. v. 47, n. 5, p. 471-474, 1998. doi: http://dx.doi.org/10.1016/S0361$\underline{9230(98) 00101-4}$

PARIETTI-WINKLER, C.; GAUCHARD, G.C.; SIMON, C.; PERRIN, P. Visual sensory preference delays balance control compensation after vestibular schwannoma surgery. Journal of Neural Neurosurgery Psychiatry. London. v.79, n. 11, p.1287-1294, 2008. doi: http://dx.doi.org/10.1136/jnnp.2007.135913

PETERKA, R. J. Sensorimotor integration in human postural control. Journal of

Neurophysiology. Bethesda. v. 88, n. 3, p. 10971118, 2002. Disponível em:

http://jn.physiology.org/content/88/3/1097.full.html. Acesso em: 23 jan. 2010.

POLLOCK, A.S.; DURWARD, B.R.; ROWE, P.J. What is balance? Clinical Reabilitation. London. v.14, n. 4, p.402-406, 2000. doi: http://dx.doi.org/10.1191/0269215500cr342oa

REASON, J.T. Motion sickness adaptation: a neural mismatch model. Journal of the Royal Society of Medicine. London. v.71, n. 11, p. 819829, 1978. Disponível em:

http://www.ncbi.nlm.nih.gov/pmc/articles/PMC143 6193/pdf//rsocmed00291-0059.pdf. Acesso em: 14 out. 2009.
RUWER, S.L.; ROSSI, A.G.; SIMON, L.F. Equilíbrio no idoso. Revista Brasileira de Otorrinolaringologia. São Paulo. v.71, n.3, p. 298-303, 2005. Disponível em: http://www.scielo.br/pdf/\%0D/rboto/v71n3/a06v71n 3.pdf. Acesso em: 14 out. 2009.

SHAHAL, B.; NACHUM, Z.; SPITZER, O.; BENDAVID, J.; DUCHMAN, H.; PODOSHIN, L.; SHUPAK, A. Computerized Dynamic Posturography and Seasickness Susceptibility. The Laryngoscope. St. Louis. v. 109, n.12B, p. 1996-2000, 1999. doi: http://dx.doi.org/10.1097/00005537-199912000$\underline{00019}$

SHUMWAY-COOK, A; HORAK, F. B. Assessing the influence of sensory interaction on balance. Suggestion from the field. Physical Therapy. Alexandria. v. 66, p. 1548-1550, 1986. Disponível em:

http://ptjournal.apta.org/content/66/10/1548.full.pdf + html. Acesso em: 19 set. 2007.

\section{Endereço:}

Danielle Bradalize

Rua XV de Novembro, 7815

Guarapuava PR Brasil

85010-000

Telefone: (42) 3622-2000

e-mail: danibranda@yahoo.com.br

Recebido em: 11 de março de 2012. Aceito em: 4 de novembro de 2012.

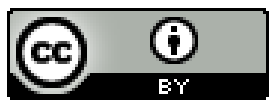

Motriz. Revista de Educação Física. UNESP, Rio Claro, SP, Brasil - elSSN: 1980-6574 - está licenciada sob Creative Commons - Atribuição 3.0 